\title{
Interpolation of Surfaces with Line of Curvature
}

\author{
Ergin Bayram*
}

(Communicated by Gudrun Albrecht)

\begin{abstract}
We introduce a method to construct parametric surfaces interpolating given finite points and a curve as a line of curvature in 3-dimensional Euclidean space. We present an existence theorem of a $C^{0}$-Hermite interpolation of surfaces possessing the given data. We show that every parameter curve of a constructed surface is a circular helix if the given curve is a circular helix. The method is validated with illustrative examples.
\end{abstract}

Keywords: $C^{0}$-Hermite interpolation, line of curvature, parametric surface.

AMS Subject Classification (2020): Primary: 53A04; Secondary: 53A05; $68 U 05$.

\section{Introduction}

A line of curvature is a surface curve whose tangent at every point is in a principal direction. This curve follows a directional flow of the extremum curvatures along the surface [11]. At a non-umblical point there are two orthogonal principal directions. A surface point is called umbilic if all normal curvatures are the same at this point. A surface curve is a line of curvature if and only if the surface normals along this curve form a developable surface [13]. A line of curvature is a crucial characteristic curve on a surface. It is a widely used tool in surface analysis for presenting variations of the principal direction. The line of curvature can guide the analysis of surfaces, widely used in Geometric Design, Shape Recognition, polygonization of surfaces and Surface Rendering $[1,9,11]$.

Curves and surfaces have been a long term research topic $[5,10,13]$. Classical theory focuses on classifying curves on a surface. However, the more interesting problem is to consider the reverse problem : the construction of surfaces possessing a given curve as a special curve. There is a vast literature on this type of construction of parametric surfaces through a given curve [2-4, 6-8, 12]. Wang et al. [12] introduced a method to obtain parametric surfaces with a common geodesic. Li et al. [8] obtained necessary and sufficient conditions for a curve to be a common line of curvature on a surface pencil. Bayram et al. [2] presented surfaces with a common asymptotic curve. Bayram [3] constructed surfaces with a common adjoint curve. Recently, Lee et al. [7] introduced a new method to construct a parametric surface in terms of curves and points placed on 3 dimensional Euclidean space.

In the present paper, we introduce interpolation of surfaces with a given line of curvature. We prove the existence of a $C^{0}$-Hermite interpolation of surfaces possessing a given curve as a line of curvature and present some illustrative examples. 


\section{Preliminaries}

Let $\alpha(s)$ be a unit speed curve framed by the so called Frenet frame $\{T(s), N(s), B(s)\}$ satisfying the following relations

$$
\left[\begin{array}{c}
T^{\prime}(s) \\
N^{\prime}(s) \\
B^{\prime}(s)
\end{array}\right]=\left[\begin{array}{ccc}
0 & \kappa(s) & 0 \\
-\kappa(s) & 0 & \tau(s) \\
0 & -\tau(s) & 0
\end{array}\right]\left[\begin{array}{c}
T(s) \\
N(s) \\
B(s)
\end{array}\right],
$$

where $\kappa(s)$ and $\tau(s)$ denotes the curvature and the torsion of $\alpha(s)$, respectively. Due to [12], surfaces possessing the curve $\alpha(s)$ can be represented parametrically as

$$
M(s, t)=\alpha(s)+f(s, t) T(s)+g(s, t) N(s)+h(s, t) B(s),
$$

where $0 \leq s \leq S, 0 \leq t \leq T$ and $f(s, t), g(s, t)$ and $h(s, t)$ are called marching-scale functions. Note that, change of marching-scale functions produces new surfaces passing through the curve $\alpha(s)$.

Definition 2.1. A curve $\alpha(s)$ on the surface $M(s, t)$ is called a parameter curve if there exists a parameter $t_{0} \in[0, T]$ such that $M\left(s, t_{0}\right)=\alpha(s)$.

Definition 2.2. Let $u$ be a unit vector tangent to $M \subset \mathbb{R}^{3}$ at a point $\mathrm{p}$ and $S$ be the shape operator of the surface. Then the number

$$
k(u)=\langle S(u), u\rangle
$$

is called the normal curvature of $M$ in the $u$ direction [10].

Definition 2.3. Let $p$ be a point of $M \subset \mathbb{R}^{3}$. The maximum and minimum values of the normal curvature $k(u)$ of $M$ at $\mathrm{p}$ are called the principal curvatures of $\mathrm{M}$ at $\mathrm{p}$. The directions in which these extreme values occur are called principal directions of $M$ at $p[10]$.

Definition 2.4. If a regular connected curve $\alpha(s)$ on $M \subset \mathbb{R}^{3}$ is such that for all $p \in \alpha$ the tangent line of $\alpha$ is a principal direction at $p$, then $\alpha$ is said to be a line of curvature of $M$ [5].

Theorem 2.1. [8] The given curve $\alpha(s)$ is a line of curvature on the surface $M(s, t)$ given by Eqn. (2.1) if and only if

$$
\left\{\begin{array}{c}
f\left(s, t_{0}\right)=g\left(s, t_{0}\right)=h\left(s, t_{0}\right) \equiv 0 \\
\phi_{1}\left(s, t_{0}\right)=0, \phi_{2}\left(s, t_{0}\right)=\lambda(s) \cos \theta, \phi_{3}\left(s, t_{0}\right)=\lambda(s) \sin \theta \\
\theta(s)=-\int_{s_{0}}^{s} \tau(u) d u+\theta_{0}
\end{array}\right.
$$

where $0 \leq s \leq S, 0 \leq t_{0} \leq T$ ( $t_{0}$ fixed $), \lambda(s) \neq 0$ and

$$
\left\{\begin{array}{c}
\phi_{1}\left(s, t_{0}\right)=\left(\frac{\partial g}{\partial s} \frac{\partial h}{\partial t}-\frac{\partial h}{\partial s} \frac{\partial g}{\partial t}\right)\left(s, t_{0}\right) \\
\phi_{2}\left(s, t_{0}\right)=\left[\frac{\partial h}{\partial s} \frac{\partial f}{\partial t}-\left(1-\frac{\partial f}{\partial s}\right) \frac{\partial h}{\partial t}\right]\left(s, t_{0}\right), \\
\phi_{3}\left(s, t_{0}\right)=\left[\left(1+\frac{\partial f}{\partial s}\right) \frac{\partial g}{\partial t}-\frac{\partial g}{\partial s} \frac{\partial f}{\partial t}\right]\left(s, t_{0}\right) .
\end{array}\right.
$$

Remark 2.1. In the last theorem, one can think the function $\lambda(s)$ as a function controlling the shape of the surface.

\section{Surface interpolation}

In this section, we obtain surfaces possessing the given curve and interpolating finite interpolation points in 3 dimensional Euclidean space.

Definition 3.1. [7] Let $P_{1}, P_{2}, \ldots, P_{n}$ be different points on $E^{3}$ and $M(s, t)$ be a surface given by Eqn. (2.1). For some different points $\left(s_{i}, t_{i}\right) \in \mathbb{R}^{2}$, we can construct the surface $M(s, t)$ such that $M\left(s_{i}, t_{i}\right)=P_{i}, i=1,2, \ldots, n$. It is called a surface interpolation associated with the given curve $\alpha(s)$ passing through $\mathrm{n}$-control points $P_{i}, i=1,2, \ldots, n$, simply, $C^{0}$-Hermite surface interpolation. In particular, $\left\{P_{1}, P_{2}, \ldots, P_{m}\right\}$ is called $C^{0}$-Hermite data.

Theorem 2.1 can be rewritten as the following. 
Corollary 3.1. The given curve $\alpha(s)$ is a line of curvature on the surface $M(s, t)$ given by Eqn. (2.1) if and only if

$$
\left\{\begin{array}{c}
f\left(s, t_{0}\right)=g\left(s, t_{0}\right)=h\left(s, t_{0}\right) \equiv 0 \\
\frac{\partial g}{\partial t}\left(s, t_{0}\right)=\lambda(s) \sin \theta, \frac{\partial h}{\partial t}\left(s, t_{0}\right)=-\lambda(s) \cos \theta \\
\theta(s)=-\int_{s_{0}}^{s} \tau(u) d u+\theta_{0}
\end{array}\right.
$$

where $0 \leq s \leq S, 0 \leq t_{0} \leq T$ ( $t_{0}$ fixed $), \lambda(s) \neq 0$.

Theorem 3.1. Let $P_{1}, P_{2}, \ldots, P_{n}$ be distinct points on $E^{3}$ and $M(s, t)$ be a surface given by Eqn. $(2.1)$. If $M\left(s_{i}, t_{i}\right)=P_{i}$, $i=1,2, \ldots, n$, then there exists a unique surface interpolation possessing the given curve as a line of curvature such that the marching-scale functions are given by

$$
\left\{\begin{array}{c}
f(s, t)=\sum_{i=1}^{n} a_{i}\left(t-t_{0}\right)^{i} \\
g(s, t)=(\sin \theta(s)) \sum_{i=1}^{n} b_{i}\left(t-t_{0}\right)^{i} \\
h(s, t)=-(\cos \theta(s)) \sum_{i=1}^{n} b_{i}\left(t-t_{0}\right)^{i} \\
\theta(s)=-\int_{s_{0}}^{s} \tau(u) d u+\theta_{0}
\end{array}\right.
$$

and

or

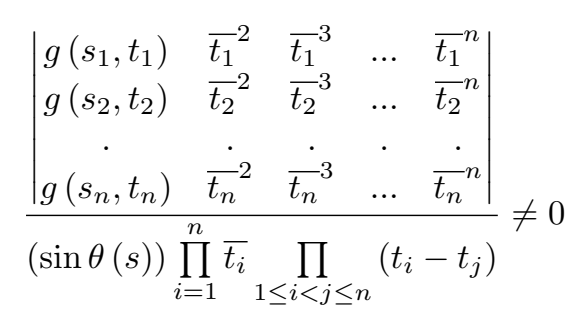

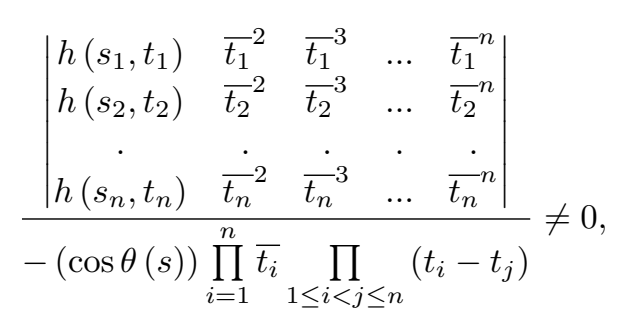

where $0 \leq s \leq S, 0 \leq t_{0} \leq T$ ( $t_{0}$ fixed $), \overline{t_{i}}=t_{i}-t_{0}, i=1,2, \ldots, n$.

Proof. Let $P_{1}, P_{2}, \ldots, P_{n}$ be distinct points on the surface $M(s, t)$ and $M\left(s_{i}, t_{i}\right)=P_{i}, i=1,2, \ldots, n$ for $0 \leq t_{0}<$ $t_{1}<t_{2}<\ldots<t_{n} \leq T$. Since

$$
M\left(s_{i}, t_{i}\right)=P_{i}=\alpha\left(s_{i}\right)+f\left(s_{i}, t_{i}\right) T(s)+g\left(s_{i}, t_{i}\right) N(s)+h\left(s_{i}, t_{i}\right) B(s),
$$

we have

$$
\left\{\begin{array}{l}
f\left(s_{i}, t_{i}\right)=\left\langle P_{i}-\alpha\left(s_{i}\right), T\left(s_{i}\right)\right\rangle \\
g\left(s_{i}, t_{i}\right)=\left\langle P_{i}-\alpha\left(s_{i}\right), N\left(s_{i}\right)\right\rangle \\
h\left(s_{i}, t_{i}\right)=\left\langle P_{i}-\alpha\left(s_{i}\right), B\left(s_{i}\right)\right\rangle
\end{array}\right.
$$

Denote $f\left(s_{i}, t_{i}\right)=c_{i 1}, g\left(s_{i}, t_{i}\right)=c_{i 2}, h\left(s_{i}, t_{i}\right)=c_{i 3}$. Letting

$$
\left\{\begin{array}{c}
f(s, t)=\sum_{i=1}^{n} a_{i}\left(t-t_{0}\right)^{i}, \\
g(s, t)=(\sin \theta(s)) \sum_{i=1}^{n} b_{i}\left(t-t_{0}\right)^{i} \\
h(s, t)=-(\cos \theta(s)) \sum_{i=1}^{n} b_{i}\left(t-t_{0}\right)^{i},
\end{array}\right.
$$

we get

$$
\left\{\begin{array}{l}
f\left(s_{i}, t_{i}\right)=a_{1}\left(t_{i}-t_{0}\right)+a_{2}\left(t_{i}-t_{0}\right)^{2}+\ldots+a_{n}\left(t_{i}-t_{0}\right)^{n}=c_{i 1} \\
g\left(s_{i}, t_{i}\right)=b_{1}\left(t_{i}-t_{0}\right)+b_{2}\left(t_{i}-t_{0}\right)^{2}+\ldots+b_{n}\left(t_{i}-t_{0}\right)^{n}=c_{i 2} \\
h\left(s_{i}, t_{i}\right)=b_{1}\left(t_{i}-t_{0}\right)+b_{2}\left(t_{i}-t_{0}\right)^{2}+\ldots+b_{n}\left(t_{i}-t_{0}\right)^{n}=c_{i 3}
\end{array}\right.
$$


$i=1,2, \ldots, n$. Denote $\overline{t_{i}}=t_{i}-t_{0}$ for $i=1,2, \ldots, n$ and obtain

$$
\begin{aligned}
& {\left[\begin{array}{cccc}
\overline{t_{1}} & {\overline{t_{1}}}^{2} & \ldots & {\overline{t_{1}}}^{n} \\
\overline{t_{2}} & {\overline{t_{2}}}^{2} & \ldots & {\overline{t_{2}}}^{n} \\
\cdot & \cdot & \cdot & \cdot \\
\overline{t_{n}} & {\overline{t_{n}}}^{2} & \ldots & {\overline{t_{n}}}^{n}
\end{array}\right]\left[\begin{array}{c}
a_{1} \\
a_{2} \\
\cdot \\
a_{n}
\end{array}\right]=\left[\begin{array}{c}
c_{11} \\
c_{21} \\
\cdot \\
c_{n 1}
\end{array}\right]} \\
& {\left[\begin{array}{cccc}
\overline{t_{1}} & {\overline{t_{1}}}^{2} & \ldots & {\overline{t_{1}}}^{n} \\
\overline{t_{2}} & {\overline{t_{2}}}^{2} & \ldots & \overline{t_{2}} \\
\dot{\cdot} & \cdot & \cdot & \cdot \\
\overline{t_{n}} & {\overline{t_{n}}}^{2} & \ldots & {\overline{t_{n}}}^{n}
\end{array}\right]\left[\begin{array}{c}
b_{1} \sin \theta(s) \\
b_{2} \sin \theta(s) \\
\cdot \\
b_{n} \sin \theta(s)
\end{array}\right]=\left[\begin{array}{c}
c_{12} \\
c_{22} \\
\cdot \\
c_{n 2}
\end{array}\right],} \\
& {\left[\begin{array}{cccc}
\overline{t_{1}} & {\overline{t_{1}}}^{2} & \ldots & \overline{t_{1}} \\
\overline{t_{2}} & {\overline{t_{2}}}^{2} & \ldots & \overline{{t_{2}}^{n}} \\
\cdot & \cdot & \cdot & \cdot \\
\overline{t_{n}} & {\overline{t_{n}}}^{2} & \ldots & {\overline{t_{n}}}^{n}
\end{array}\right]\left[\begin{array}{c}
-b_{1} \cos \theta(s) \\
-b_{2} \cos \theta(s) \\
\cdot \\
-b_{n} \cos \theta(s)
\end{array}\right]=\left[\begin{array}{c}
c_{13} \\
c_{23} \\
\cdot \\
c_{n 3}
\end{array}\right]}
\end{aligned}
$$

Since $t_{i} \neq t_{j}$ for $1 \leq i<j \leq n$ and $t_{i} \neq 0, t_{j} \neq 0$ for $i, j=1,2, \ldots, n$

$$
\operatorname{det}\left[\begin{array}{cccc}
\overline{t_{1}} & {\overline{t_{1}}}^{2} & \ldots & {\overline{t_{1}}}^{n} \\
\overline{t_{2}} & {\overline{t_{2}}}^{2} & \ldots & {\overline{t_{2}}}^{n} \\
\cdot & \cdot & \cdot & \cdot \\
\overline{t_{n}} & {\overline{t_{n}}}^{2} & \ldots & {\overline{t_{n}}}^{n}
\end{array}\right] \neq 0
$$

which implies $a_{i}, b_{i}$ have unique solutions. Eqns. (3.1) and (3.2) quarantees the regularity of the surface given by Eqn. (2.1) along the given curve $\alpha(s)$. As a result, there exists a unique surface interpolation passing through the distinct points $P_{1}, P_{2}, \ldots, P_{n}$ and possessing the given curve as a line of curvature.

Theorem 3.2. Let a unit speed circular helix $\alpha(s)$ be a line of curvature on the parametric surface given by Eqn. (2.1) and assume

$$
\left\{\begin{array}{c}
f(s, t)=\sum_{i=1}^{n} a_{i}\left(t-t_{0}\right)^{i}, \\
g(s, t)=(\sin \theta(s)) \sum_{i=1}^{n} b_{i}\left(t-t_{0}\right)^{i}, \\
h(s, t)=-(\cos \theta(s)) \sum_{i=1}^{n} b_{i}\left(t-t_{0}\right)^{i} \\
\theta(s)=-\int_{s_{0}}^{s} \tau(u) d u+\theta_{0},
\end{array}\right.
$$

$0 \leq s \leq S, 0 \leq t \leq T$. For a constant $\overline{t_{0}} \in[0, T]$, a curve $\bar{\alpha}(s)=M\left(s, \overline{t_{0}}\right)$ on the surface $M(s, t)$ is also a circular helix.

Proof. Assume that the conditions of the theorem are satisfied. We write

$$
\bar{\alpha}(s)=M\left(s, \overline{t_{0}}\right)=\alpha(s)+f\left(s, \overline{t_{0}}\right) T(s)+g\left(s, \overline{t_{0}}\right) N(s)+h\left(s, \overline{t_{0}}\right) B(s)
$$

and calculate

$$
\left\{\begin{array}{c}
\bar{\alpha}^{\prime}(s)=c_{1} T(s)+c_{2} N(s), \\
\bar{\alpha}^{\prime \prime}(s)=d_{1} T(s)+d_{2} N(s)+d_{3} B(s), \\
\bar{\alpha}^{\prime \prime \prime}(s)=e_{1} T(s)+e_{2} N(s)+e_{3} B(s),
\end{array}\right.
$$

where $c_{1}, c_{2}, d_{1}, d_{2}, d_{3}, e_{1}, e_{2}, e_{3}$ are constants, implying that the curvature and the torsion of $\bar{\alpha}(s)$ is constant and it is also a circular helix on the surface $M(s, t)$. 


\section{Examples}

\subsection{Example}

Consider the unit speed curve $\alpha(s)=\left(\cos \left(\frac{\sqrt{2}}{2} s\right), \sin \left(\frac{\sqrt{2}}{2} s\right), \frac{\sqrt{2}}{2} s\right)$ framed by

$$
\left\{\begin{array}{c}
T(s)=\frac{\sqrt{2}}{2}\left(-\sin \left(\frac{\sqrt{2}}{2} s\right), \cos \left(\frac{\sqrt{2}}{2} s\right), \frac{\sqrt{2}}{2}\right) \\
N(s)=\left(-\cos \left(\frac{\sqrt{2}}{2} s\right),-\sin \left(\frac{\sqrt{2}}{2} s\right), 0\right) \\
B(s)=\frac{\sqrt{2}}{2}\left(\sin \left(\frac{\sqrt{2}}{2} s\right),-\cos \left(\frac{\sqrt{2}}{2} s\right), 1\right)
\end{array}\right.
$$

$0 \leq s \leq 9$, with torsion $\tau(s)=\frac{1}{2}$. Given the point $P_{1}(1,2,3)$ we will obtain a surface that interpolates $\alpha(s)$ as a line of curvature and $P$ is a point on this surface. Assume $t_{0}=0, M(0,1)=P_{1}(1,2,3)$, that is, $s_{1}=0, t_{1}=1$. Letting $s_{0}=0$ we have $\theta(s)=-\frac{s}{2}$. Choosing marching-scale functions as

$$
f(s, t)=a_{1} t, g(s, t)=-b_{1} t \sin \frac{s}{2}, h(s, t)=-b_{1} t \cos \frac{s}{2}
$$

we obtain $a_{1}=\frac{5 \sqrt{2}}{2}, b_{1}=-\frac{\sqrt{2}}{2}$ and

$$
f(s, t)=\frac{5 \sqrt{2}}{2} t, g(s, t)=\frac{\sqrt{2}}{2} t \sin \frac{s}{2}, h(s, t)=\frac{\sqrt{2}}{2} t \cos \frac{s}{2} .
$$

Thus, we construct the surface

$$
\begin{aligned}
M_{1}(s, t)= & \left(\left(1-\frac{\sqrt{2}}{2} t \sin \frac{s}{2}\right) \cos \left(\frac{\sqrt{2}}{2} s\right)+\frac{t}{2}\left(\cos \frac{s}{2}-5\right) \sin \left(\frac{\sqrt{2}}{2} s\right),\right. \\
& \left(1-\frac{\sqrt{2}}{2} t \sin \frac{s}{2}\right) \sin \left(\frac{\sqrt{2}}{2} s\right)+\frac{t}{2}\left(5-\cos \frac{s}{2}\right) \cos \left(\frac{\sqrt{2}}{2} s\right), \\
& \left.\frac{\sqrt{2}}{2} s+\frac{t}{2}\left(5+\cos \frac{s}{2}\right)\right),
\end{aligned}
$$

$0 \leq s \leq 9, \quad 0 \leq t \leq 2$, that interpolates $\alpha(s)$ as a line of curvature and $P_{1}$ is a point on this surface (Fig. 1).

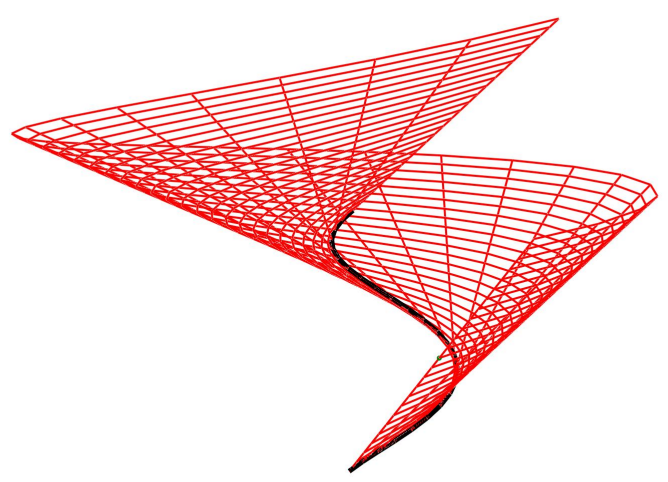

Figure 1. The surface $M_{1}(s, t)$ passing through the point $P_{1}(1,2,3)$ (green in color) and possessing the given curve $\alpha(s)$ as a line of curvature (black in color).

Now, we will find another surface passing through the points $P_{1}(1,2,3), P_{2}(1,4,5)$ and interpolating the curve $\alpha(s)$ as a line of curvature. Choosing $s_{1}=s_{2}=0, t_{1}=1$ and $t_{2}=2$, we have

$$
f(s, t)=a_{1} t+a_{2} t^{2}, g(s, t)=-\left(b_{1} t+b_{2} t^{2}\right) \sin \frac{s}{2}, h(s, t)=-\left(b_{1} t+b_{2} t^{2}\right) \cos \frac{s}{2}
$$


and calculate $a_{1}=\frac{11 \sqrt{2}}{4}, a_{2}=\frac{-\sqrt{2}}{4}, b_{1}=-\frac{3 \sqrt{2}}{4}, b_{2}=\frac{\sqrt{2}}{4}$ yielding

$$
\left\{\begin{array}{c}
f(s, t)=\frac{11 \sqrt{2}}{4} t-\frac{\sqrt{2}}{4} t^{2}, g(s, t)=\left(\frac{3 \sqrt{2}}{4} t-\frac{\sqrt{2}}{4} t^{2}\right) \sin \frac{s}{2}, \\
h(s, t)=\left(\frac{3 \sqrt{2}}{4} t-\frac{\sqrt{2}}{4} t^{2}\right) \cos \frac{s}{2} .
\end{array}\right.
$$

Hence, we obtain the surface

$$
\begin{aligned}
M_{2}(s, t)= & \left(\left(1+\left(\frac{\sqrt{2}}{4} t^{2}-\frac{3 \sqrt{2}}{4} t\right) \sin \frac{s}{2}\right) \cos \left(\frac{\sqrt{2}}{2} s\right)\right. \\
& +\left(\frac{t^{2}}{4}-\frac{11 t}{4}+\left(\frac{3 t}{4}-\frac{t^{2}}{4}\right) \cos \frac{s}{2}\right) \sin \left(\frac{\sqrt{2}}{2} s\right), \\
& \left(1+\left(\frac{\sqrt{2}}{4} t^{2}-\frac{3 \sqrt{2}}{4} t\right) \sin \frac{s}{2}\right) \sin \left(\frac{\sqrt{2}}{2} s\right) \\
& +\left(\frac{11 t}{4}-\frac{t^{2}}{4}+\left(\frac{t^{2}}{4}-\frac{3 t}{4}\right) \cos \frac{s}{2}\right) \cos \left(\frac{\sqrt{2}}{2} s\right), \\
& \left.\frac{\sqrt{2}}{2} s+\frac{11 t}{4}-\frac{t^{2}}{4}+\left(\frac{3 t}{4}-\frac{t^{2}}{4}\right) \cos \frac{s}{2}\right),
\end{aligned}
$$

$0 \leq s \leq 9, \quad 0 \leq t \leq 4$ that interpolates $\alpha(s)$ as a line of curvature and $P_{1}$ and $P_{2}$ are points on this surface (Fig. 2).

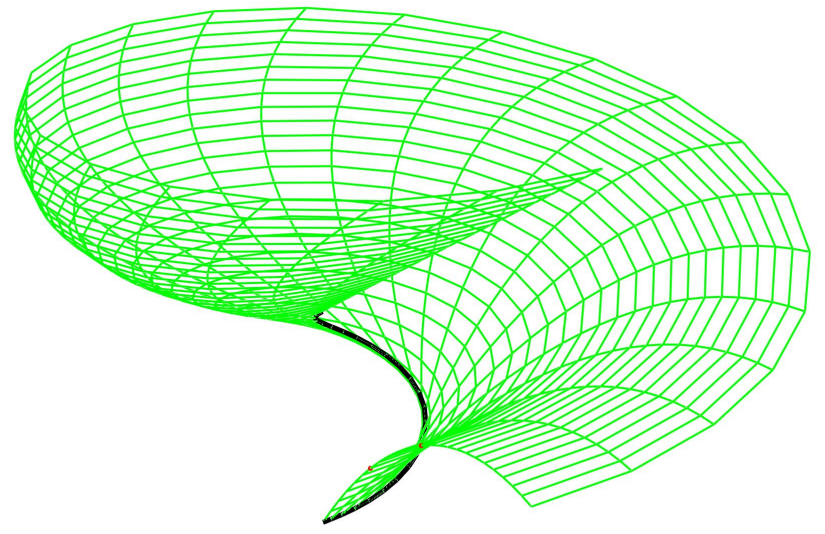

Figure 2. The surface $M_{2}(s, t)$ passing through the points $P_{1}(2,1,3), P_{2}(3,4,5)$ (red in color) and possessing the given curve $\alpha(s)$ as a line of curvature (black in color).

Example 4.1. Let us consider the circle $\alpha(s)=(\cos s, \sin s, 0)$. Its Frenet frame is

$$
\left\{\begin{array}{c}
T(s)=(-\sin s, \cos , 0) \\
N(s)=(-\cos s,-\sin s, 0), \\
B(s)=(0,0,1)
\end{array}\right.
$$

and torsion is $\tau(s)=0$ since it is a plane curve. Observe that $\theta(s)=0$. We will construct a surface interpolating the curve $\alpha(s)$ as a line of curvature and the point $P_{1}(1,1,1)$. Assume that $t_{0}=0, s_{1}=0$ and $t_{1}=1$, that is $M(0,1)=P_{1}(1,1,1)$. We calculate $f(s, t)=h(s, t)=t$ and $g(s, t) \equiv 0$. Hence, we obtain

$$
\begin{aligned}
M_{3}(s, t) & =\alpha(s)+t T(s)+t B(s) \\
& =(\cos s-t \sin s, \sin s+t \cos s, t),
\end{aligned}
$$

where $0 \leq s \leq 2 \pi,-5 \leq t \leq 5$ (Figure 3 ). 


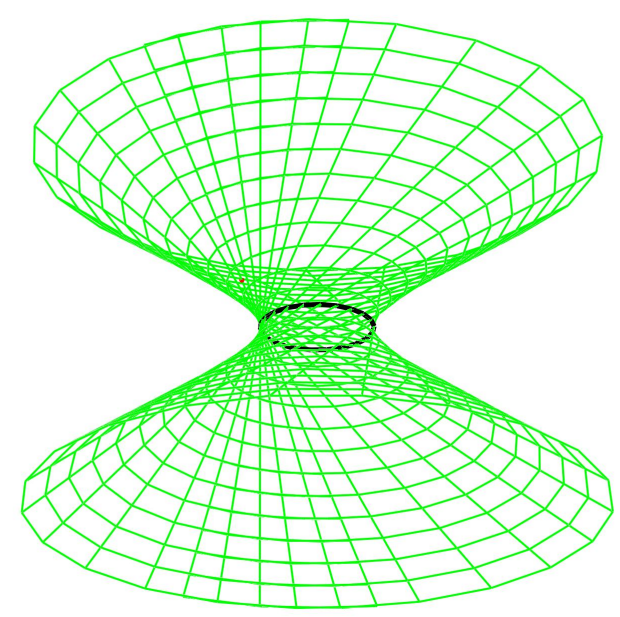

Figure 3. The surface $M_{3}(s, t)$ passing through the point $P_{1}(1,1,1)$ (red in color) and possessing the given curve $\alpha(s)$ as a line of curvature (black in color).

Now, using the same curve we construct another surface interpolating $\alpha(s)$ as a line of curvature and passing through the points $P_{1}(1,1,1)$ and $P_{2}(1,2,4)$. Suppose now $s_{1}=s_{2}=0$ and $t_{1}=1, t_{2}=2$. After a simple computation we obtain $f(s, t)=t, g(s, t) \equiv 0$ and $h(s, t)=t^{2}$. As a result, we obtain the surface

$$
\begin{aligned}
M_{4}(s, t) & =\alpha(s)+t T(s)+t^{2} B(s) \\
& =\left(\cos s-t \sin s, \sin s+t \cos s, t^{2}\right),
\end{aligned}
$$

where $0 \leq s \leq 2 \pi,-5 \leq t \leq 5$ interpolating $\alpha(s)$ as a line of curvature and passing through the points $P_{1}(1,1,1)$ and $P_{2}(1,2,4)$ (Figure 4 ).

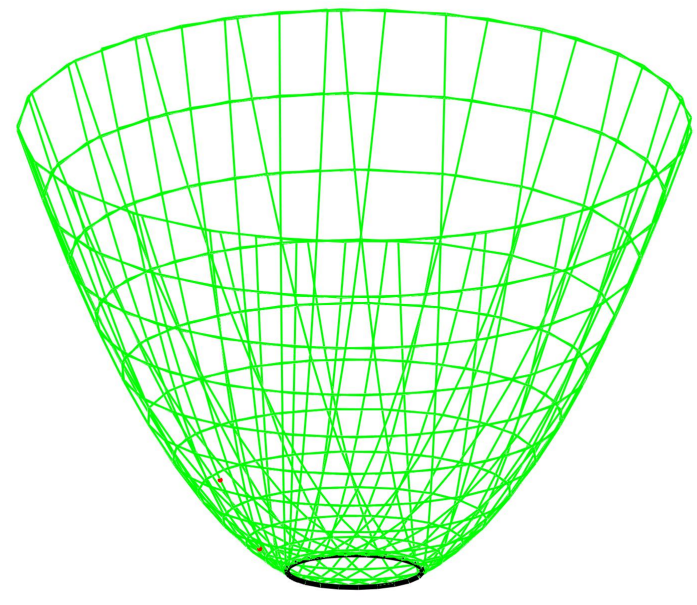

Figure 4. The surface $M_{4}(s, t)$ passing through the points $P_{1}(1,1,1), P_{2}(1,2,4)$ (red in color) and possessing the given curve $\alpha(s)$ as a line of curvature (black in color). 


\section{Conclusion}

The present study introduces a method to construct parametric surfaces interpolating given finite points and a curve as a line of curvature in 3-dimensional Euclidean space. An existence theorem of a $C^{0}$-Hermite interpolation of surfaces possessing the given data is expressed. For future studies, there are several open problems. For example, what happens if two or more surfaces are to be connected? Another possibilty is to consider to construct surfaces with Bézier, B-Spline or NURBS form for the line of curvature and for the sweeping blending functions.

\section{Acknowledgments}

The author would like to present his appreciation to the Editor and the Referees. Their suggestions help to improve the clearity of the manuscript.

\section{References}

[1] Alliez P., Cohen-Steiner D., Devillers O., Lévy B., Desbrum M. Anisotropic polygonal pemeshing. In: Proceeding of ACM SIGGRAPH.485-493 (2003).

[2] Bayram E., Güler F., Kasap E.: Parametric representation of a surface pencil with a common asymptotic curve. Computer Aided Design. 44, 637-643 (2012).

[3] Bayram E.: Surface pencil with a common adjoint curve. Turkish Journal of Mathematics. 44, 1649-1659 (2020).

[4] Bayram E., Ergün E., Kasap E.: Surface family with a common natural asymptotic lift. Journal of Science and Arts. 2, 117-124 (2015).

[5] do Carmo M. P.: Differential geometry of curves and surfaces. Prentice-Hall Inc., Englewood Cliffs, New Jersey (1976).

[6] Güler F., Bayram E., Kasap E.: Offset surface pencil with a common asymptotic curve. International Journal of Geometric Methods in Modern Physics. 15 (11), 1850195 (2018).

[7] Lee C. L., Lee J. W., Yoon D. W.: Interpolation of surfaces with geodesic. Journal of the Korean Mathematical Society. 57 (4), $957-971$ (2020).

[8] Li C. Y., Wang R. H., Zhu C. G.: Parametric representation of a surface pencil with a common line of curvature. Computer Aided Design. 43, 1110-1117 (2011).

[9] Maekawa T., Wolter F. E., Patrikalakis N. M.: Umbilics and lines of curvature for shape interrogation. Computer Aided Geometric Design. 13 (2), 133-161 (1996).

[10] O'Neill B.: Elementary differential geometry. Elsevier Inc., (1966).

[11] Patrikalakis N. M., Maekawa T.: Shape interrogation for computer aided design and manufacturing. Springer-Verlag, Heidelberg (2002).

[12] Wang G.J., Tang K., Tai C.L.: Parametric representation of a surface pencil with a common spatial geodesic. Computer Aided Design. 36, 447-459 (2004)..

[13] Willmore T. J.: An introduction to differential geometry. Dover Publications (2012).

\section{Affiliations}

\section{ERGIN BAYRAM}

ADDRESS: Ondokuz Mayis University, Dept. of Mathematics, 55270, Samsun-Turkey.

E-MAIL: erginbayram@yahoo.com

ORCID ID: 0000-0003-2633-0991 